OPEN ACCESS

Edited by:

Mikhail Lebedev,

Duke University, United States

Reviewed by:

Thomas E. Nichols,

Independent Researcher, Oxford, United Kingdom

Livio Finos,

Università degli Studi di Padova, Italy Robin A. A. Ince,

University of Manchester, United Kingdom

*Correspondence: Andy W. K. Yeung ndyeung@hku.hk

Received: 02 May 2017 Accepted: 12 January 2018 Published: 26 January 2018

Citation:

Yeung AWK (2018) An Updated Survey on Statistical Thresholding and Sample Size of fMRI Studies.

Front. Hum. Neurosci. 12:16. doi: 10.3389/fnhum.2018.00016

\section{An Updated Survey on Statistical Thresholding and Sample Size of fMRI Studies}

\author{
Andy W. K. Yeung*
}

Oral and Maxillofacial Radiology, Applied Oral Sciences, Faculty of Dentistry, The University of Hong Kong, Pok Fu Lam, Hong Kong

Background: Since the early 2010s, the neuroimaging field has paid more attention to the issue of false positives. Several journals have issued guidelines regarding statistical thresholds. Three papers have reported the statistical analysis of the thresholds used in $\mathrm{fMRI}$ literature, but they were published at least 3 years ago and surveyed papers published during 2007-2012. This study revisited this topic to evaluate the changes in this field.

Methods: The PubMed database was searched to identify the task-based (not resting-state) fMRI papers published in 2017 and record their sample sizes, inferential methods (e.g., voxelwise or clusterwise), theoretical methods (e.g., parametric or non-parametric), significance level, cluster-defining primary threshold (CDT), volume of analysis (whole brain or region of interest) and software used.

Results: The majority (95.6\%) of the 388 analyzed articles reported statistics corrected for multiple comparisons. A large proportion (69.6\%) of the 388 articles reported main results by clusterwise inference. The analyzed articles mostly used software Statistical Parametric Mapping (SPM), Analysis of Functional Neurolmages (AFNI), or FMRIB Software Library (FSL) to conduct statistical analysis. There were $70.9 \%, 37.6 \%$, and $23.1 \%$ of SPM, AFNI, and FSL studies, respectively, that used a CDT of $p \leq 0.001$. The statistical sample size across the articles ranged between 7 and 1,299 with a median of 33. Sample size did not significantly correlate with the level of statistical threshold.

Conclusion: There were still around 53\% (142/270) studies using clusterwise inference that chose a more liberal CDT than $p=0.001(n=121)$ or did not report their CDT $(n=21)$, down from around $61 \%$ reported by Woo et al. (2014). For FSL studies, it seemed that the CDT practice had no improvement since the survey by Woo et al. (2014). A few studies chose unconventional CDT such as $p=0.0125$ or 0.004 . 
Such practice might create an impression that the threshold alterations were attempted to show "desired" clusters. The median sample size used in the analyzed articles was similar to those reported in previous surveys. In conclusion, there seemed to be no change in the statistical practice compared to the early 2010 s.

Keywords: false-discovery rate, familywise error rate, fMRI, Gaussian random field, literature, Monte Carlo stimulation, threshold, threshold-free cluster enhancement

\section{INTRODUCTION}

Functional magnetic resonance imaging (fMRI) studiesparticularly the task-based fMRI studies, the most popular type of fMRI study-enable researchers to examine the human brain about various aspects ranging from sensation to cognition. Findings may bear clinical relevance such as the identification of neural correlates of diseases or the enabling of a neuro-functional assessment of clinical treatments.

The reproducibility of a neuroscience report depends on numerous factors-including the methodological details, statistical power and flexibility of the analyses (Carp, 2012). One of the most important factors that could be assessed relatively easily is the statistical approach used. Every paper may set its own significance level for the statistical tests reported (Hupé, 2015), and therefore, one may need to interpret the significant results from different papers differently. Considering the mass-univariate analytic approach utilized by various popular fMRI data-processing software-such as Statistical Parametric Mapping (SPM) (Penny et al., 2011), Analysis of Functional NeuroImages (AFNI) (Cox, 1996), and FMRIB Software Library (FSL) (Jenkinson et al., 2012) -correction for multiple comparisons is crucial for simultaneous statistical tests on several thousands of voxels. With regard to proper corrections for multiple comparisons, Carp (2012) revealed that an astonishing $41 \%$ of his 241 surveyed studies, which were published during 2007-2012, did not report formal corrections. As an extension to his work, Guo et al. (2014) reported a much reduced 19\% for their 100 surveyed studies, which were published in six leading neuroscience/neuroimaging/multidisciplinary journals during 2010-2011. Similarly, Woo et al. (2014) reported that $6 \%$ of their 814 surveyed studies, which were published in seven leading journals during 2010-2011, did not apply formal statistical corrections. Uncorrected results may contain high false-positive rates, and therefore, their reproducibility and clinical relevance could potentially be undermined. Even for corrected results, the improper setting of statistical thresholds may also lead to inflated false-positive rates. Woo et al. (2014) and Eklund et al. (2016) have repeatedly stated that routine voxelwise correction methods are adequate for controlling false positives whereas cluster-defining primary thresholds (CDT) for clusterwise inferences should be set at $p=0.001$ or lower because more liberal thresholds, such as $p=0.01$, may cause highly inflated false-positive rates for parametric methods. Clusterwise inference was the most popular method because it is more sensitive when detecting significance (i.e., more powerful); however, its spatial precision is inferior to that of voxelwise inference, as a large significant cluster can only indicate that significant activations are contained within the cluster.
Clusterwise inference gives no information with regard to which voxels are significantly activated (Woo et al., 2014).

In 2016, two journals issued guidelines regarding their stance on the standard statistical thresholds of reported fMRI/neuroimaging results (Carter et al., 2016; Roiser et al., 2016). Table 1 lists the key points of these guidelines and the suggestions of Woo et al. (2014) and Eklund et al. (2016). Moreover, several years have lapsed since 2014, the year when the last survey was published (Guo et al., 2014). It is time to conduct a literature survey on the statistical thresholds used by the fMRI studies published most recently.

\section{MATERIALS AND METHODS}

In accordance with the methods of previous studies (Carp, 2012; Guo et al., 2014), articles published in 2017 and written in English were identified with the keywords "fMRI," "BOLD," and "task" in the PubMed database. The search was performed on July 20, 2017. These criteria yielded 1,020 articles (listed in Supplementary File S1). For this study, all 1,020 articles were initially included, and each was assessed by reading its full text and excluded if it did not report task-based human fMRI studies and did not report results from SPM. In other words, studies that reported animal studies, resting-state fMRI, connectivity, multivoxel pattern analysis or percent of signal change were excluded. The screening excluded 632 articles accordingly and finally a total of 388 articles entered the analysis (Supplementary File S1). For the 388 articles, items including sample size, inferential method (e.g., voxelwise or clusterwise), theoretical method of correction for multiple comparisons (e.g., parametric or non-parametric), significance level, CDT (if applicable), volume of analysis (whole brain or region of interest; ROI) and software used were recorded manually. For articles that used multiple thresholds, the most stringent one used for the main analyses was chosen (Woo et al., 2014). Pearson's correlation test was performed to evaluate the relationship between the sample size and the levels of CDT in the articles using clusterwise inference.

\section{RESULTS}

\section{Sample Size and Software Used}

The sample size reported in 388 papers ranged from 7 to 1,299 with a median of 33 . One hundred and thirty-eight studies (35.6\%) analyzed data from 25 or fewer subjects, 152 studies (39.2\%) had 26-50 subjects, 54 studies (13.9\%) had 51-75 subjects, 23 studies (5.9\%) had 76-100 subjects and 21 studies (5.4\%) had 101 or more subjects (Figure 1). 
TABLE 1 | Recently published recommended statistical practices for controlling false positives.

\begin{tabular}{|c|c|}
\hline Publication name & Recommendations \\
\hline Woo et al., 2014 & $\begin{array}{l}\text { 1. Set the default cluster-defining primary threshold (CDT) at } p<0.001 .2 \text {. Use a stringent CDT or voxelwise inference for highly powered } \\
\text { studies. }\end{array}$ \\
\hline Eklund et al., 2016 & $\begin{array}{l}\text { 1. The parametric method works well for voxelwise inferences but not for clusterwise inferences (unless a stringent CDT is set at } p<0.001 \text { ). } \\
\text { 2. The permutation method works well for both voxelwise and clusterwise inferences. }\end{array}$ \\
\hline Roiser et al., 2016 & $\begin{array}{l}\text { 1. For clusterwise inferences, choose a stringent CDT (e.g., } p<0.001) \text { unless the permutation method was employed. } 2 \text {. For voxelwise } \\
\text { inferences, } p \text {-values should be corrected for multiple comparisons. 3. Complementary approaches, such as false-discovery rate or } \\
\text { threshold-free cluster enhancement, can be considered. } 4 \text {. Preregister the proposed studies in which the planned statistical analyses } \\
\text { methods are documented clearly. }\end{array}$ \\
\hline Carter et al., 2016 & $\begin{array}{l}\text { 1. Studies investigating very small brain regions should use a high voxel threshold (e.g., } p<0.001) .2 \text {. Studies not targeting precise } \\
\text { localization may consider a more liberal threshold and focus on controlling false negatives by data reduction (e.g., region-of-interest } \\
\text { analyses), as studies with fewer than } 50 \text { subjects per group usually have limited power. }\end{array}$ \\
\hline
\end{tabular}

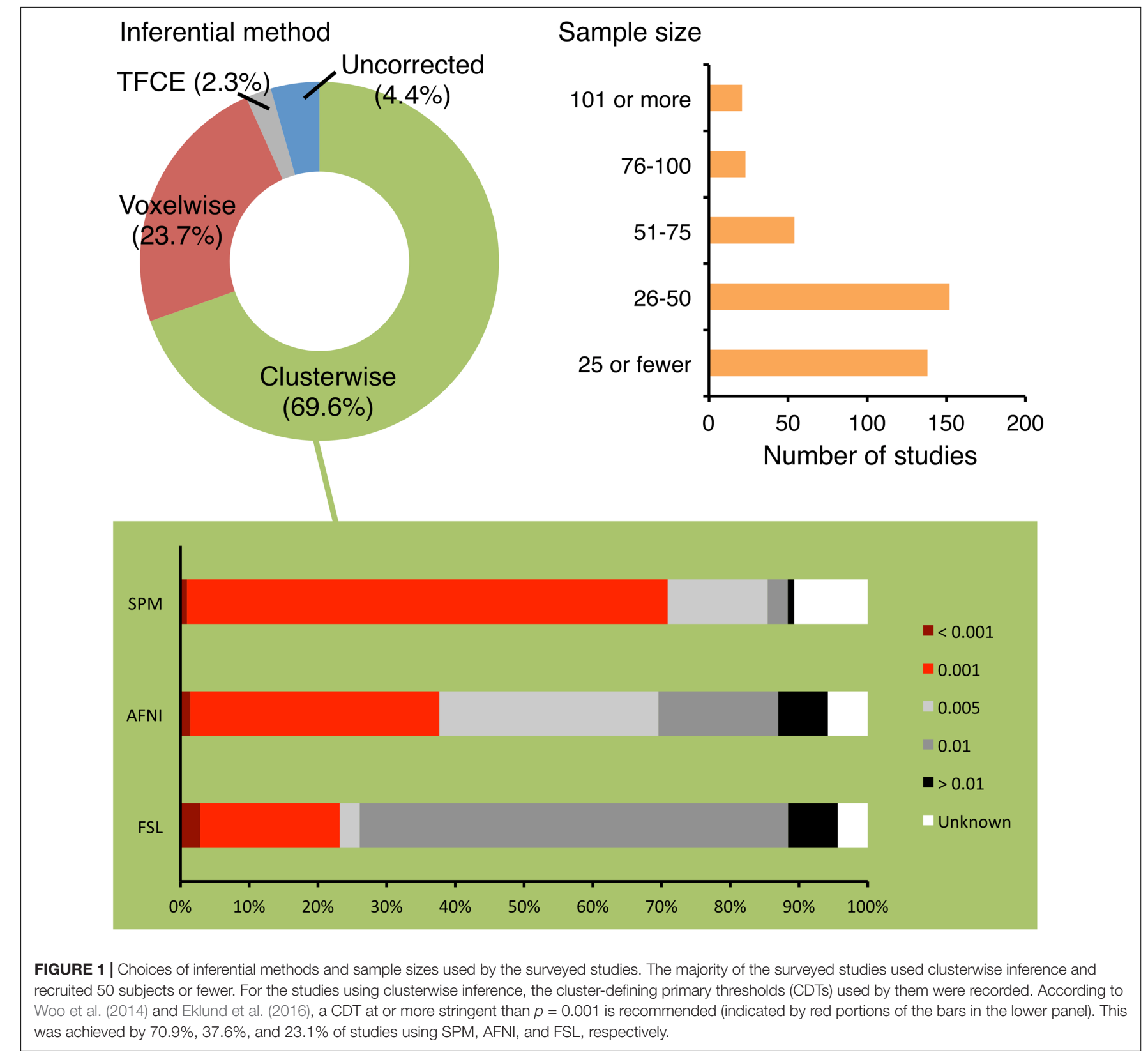


TABLE 2 | The 125 journals that published the 388 analyzed articles.

\begin{tabular}{|c|c|c|c|c|c|}
\hline Journal list & Count & $\%$ & Journal list (continued) & Count & $\%$ \\
\hline Neuroimage & 23 & 5.9 & Alzheimers Dement (Amst) & 1 & 0.3 \\
\hline Cortex & 14 & 3.6 & Appl Neuropsychol Child & 1 & 0.3 \\
\hline Neuropsychologia & 14 & 3.6 & Arch Gerontol Geriatr & 1 & 0.3 \\
\hline Brain Imaging Behav & 13 & 3.4 & Behav Res Ther & 1 & 0.3 \\
\hline Cereb Cortex & 13 & 3.4 & BMC Psychiatry & 1 & 0.3 \\
\hline Hum Brain Mapp & 13 & 3.4 & Br J Psychiatry & 1 & 0.3 \\
\hline J Neurosci & 12 & 3.1 & Br J Sports Med & 1 & 0.3 \\
\hline Plos One & 12 & 3.1 & Cerebellum & 1 & 0.3 \\
\hline Sci Rep & 12 & 3.1 & Cogn Neurosci & 1 & 0.3 \\
\hline J Cogn Neurosci & 11 & 2.8 & Cultur Divers Ethnic Minor Psychol & 1 & 0.3 \\
\hline Behav Brain Res & 10 & 2.6 & Dev Psychol & 1 & 0.3 \\
\hline Psychiatry Res & 9 & 2.3 & Einstein (Sao Paulo) & 1 & 0.3 \\
\hline J Affect Disord & 8 & 2.1 & Emotion & 1 & 0.3 \\
\hline Neuroimage Clin & 8 & 2.1 & Epilepsy Behav & 1 & 0.3 \\
\hline Brain Struct Funct & 6 & 1.5 & Eur Child Adolesc Psychiatry & 1 & 0.3 \\
\hline Neuroscience & 6 & 1.5 & Eur Eat Disord Rev & 1 & 0.3 \\
\hline Soc Cogn Affect Neurosci & 6 & 1.5 & Eur J Paediatr Neurol & 1 & 0.3 \\
\hline Addict Biol & 5 & 1.3 & Eur J Pain & 1 & 0.3 \\
\hline Biol Psychol & 5 & 1.3 & Eur Neuropsychopharmacol & 1 & 0.3 \\
\hline Dev Psychopathol & 5 & 1.3 & Eur Radiol & 1 & 0.3 \\
\hline Neuropsychopharmacology & 5 & 1.3 & Front Aging Neurosci & 1 & 0.3 \\
\hline Psychol Med & 5 & 1.3 & Front Neuroanat & 1 & 0.3 \\
\hline Soc Neurosci & 5 & 1.3 & Front Psychol & 1 & 0.3 \\
\hline Addiction & 4 & 1 & Int J Neuropsychopharmacol & 1 & 0.3 \\
\hline Brain Cogn & 4 & 1 & Int J Neurosci & 1 & 0.3 \\
\hline Brain Res & 4 & 1 & Int J Psychophysiol & 1 & 0.3 \\
\hline Cogn Affect Behav Neurosci & 4 & 1 & J Alzheimers Dis & 1 & 0.3 \\
\hline Dev Sci & 4 & 1 & J Am Acad Child Adolesc Psychiatry & 1 & 0.3 \\
\hline Eur J Neurosci & 4 & 1 & J Atten Disord & 1 & 0.3 \\
\hline Front Behav Neurosci & 4 & 1 & J Autism Dev Disord & 1 & 0.3 \\
\hline Front Hum Neurosci & 4 & 1 & J Child Sex Abus & 1 & 0.3 \\
\hline Mult Scler & 4 & 1 & J Clin Exp Neuropsychol & 1 & 0.3 \\
\hline Transl Psychiatry & 4 & 1 & J Hypertens & 1 & 0.3 \\
\hline Biol Psychiatry & 3 & 0.8 & J Neurol Neurosurg Psychiatry & 1 & 0.3 \\
\hline Brain Behav & 3 & 0.8 & J Neuropsychol & 1 & 0.3 \\
\hline Brain Lang & 3 & 0.8 & J Neurotrauma & 1 & 0.3 \\
\hline Elife & 3 & 0.8 & J Orthop Sports Phys Ther & 1 & 0.3 \\
\hline Eur Arch Psychiatry Clin Neurosci & 3 & 0.8 & J Physiol Anthropol & 1 & 0.3 \\
\hline Neural Plast & 3 & 0.8 & J Psycholinguist Res & 1 & 0.3 \\
\hline Psychopharmacology (Berl) & 3 & 0.8 & J Speech Lang Hear Res & 1 & 0.3 \\
\hline Schizophr Res & 3 & 0.8 & J Vis Exp & 1 & 0.3 \\
\hline Alcohol Alcohol & 2 & 0.5 & J Voice & 1 & 0.3 \\
\hline Am J Psychiatry & 2 & 0.5 & JAMA Psychiatry & 1 & 0.3 \\
\hline Bipolar Disord & 2 & 0.5 & Nat Commun & 1 & 0.3 \\
\hline Brain Stimul & 2 & 0.5 & Neural Regen Res & 1 & 0.3 \\
\hline Brain Topogr & 2 & 0.5 & Neurobiol Learn Mem & 1 & 0.3 \\
\hline Brain & 2 & 0.5 & Neurodegener Dis & 1 & 0.3 \\
\hline Clin Physiol Funct Imaging & 2 & 0.5 & Neurogastroenterol Motil & 1 & 0.3 \\
\hline Depress Anxiety & 2 & 0.5 & Neurol Med Chir (Tokyo) & 1 & 0.3 \\
\hline Dev Cogn Neurosci & 2 & 0.5 & Neurology & 1 & 0.3 \\
\hline Drug Alcohol Depend & 2 & 0.5 & Neuropsychobiology & 1 & 0.3 \\
\hline Exp Brain Res & 2 & 0.5 & Neuroradiology & 1 & 0.3 \\
\hline
\end{tabular}


TABLE 2 | Continued

\begin{tabular}{|c|c|c|c|c|c|}
\hline Journal list & Count & $\%$ & Journal list (continued) & Count & $\%$ \\
\hline Hippocampus & 2 & 0.5 & Nutr Neurosci & 1 & 0.3 \\
\hline J Int Neuropsychol Soc & 2 & 0.5 & Obes Res Clin Pract & 1 & 0.3 \\
\hline J Psychiatr Res & 2 & 0.5 & Physiol Rep & 1 & 0.3 \\
\hline J Psychopharmacol & 2 & 0.5 & PLoS Biol & 1 & 0.3 \\
\hline Mol Psychiatry & 2 & 0.5 & Proc IEEE Inst Electr Electron Eng & 1 & 0.3 \\
\hline Neurobiol Aging & 2 & 0.5 & Psychiatry Clin Neurosci & 1 & 0.3 \\
\hline Proc Natl Acad Sci USA & 2 & 0.5 & Psychophysiology & 1 & 0.3 \\
\hline Prog Neuropsychopharmacol Biol Psychiatry & 2 & 0.5 & Res Dev Disabil & 1 & 0.3 \\
\hline Psychoneuroendocrinology & 2 & 0.5 & Schizophr Bull & 1 & 0.3 \\
\hline Acta Radiol & 1 & 0.3 & Swiss Med Wkly & 1 & 0.3 \\
\hline Alcohol Clin Exp Res & 1 & 0.3 & & & \\
\hline
\end{tabular}

The studies were published in 125 journals (Table 2). The studies predominantly used SPM for statistical analyses (202, 52.1\%)-followed by FSL (79, 20.4\%), AFNI (71, $18.3 \%)$, BrainVoyager $(11,2.8 \%)$, Resting-State fMRI Data Analysis Toolkit (6, 1.5\%), Statistical Non-Parametric Mapping (SnPM; 5, 1.3\%), and Matlab but other toolbox than SPM or SnPM $(5,1.3 \%)$. There was one study that used FreeSurfer, one used MAsks for Region of INterest Analysis, one used FIDL (developed by Washington University in St. Louis), one used TFCE toolbox (University of Jena) and one used XBAM (developed by King's College London).

\section{Choice of Inferential Method, Theoretical Method, and Significance Level}

The majority of studies $(371,95.6 \%)$ reported main results with statistics corrected for multiple comparisons. Of the analyzed studies, 270 (69.6\%) reported clusterwise inference for their main analyses whereas $92(23.7 \%)$ reported using voxelwise inference and nine $(2.3 \%)$ reported using the thresholdfree cluster enhancement (TFCE) inference (Figure 1). Most of the studies defined significance at corrected $p=0.05$. There were 338 studies $(87.1 \%)$ that reported whole-brain results for their main analyses and 244 of them (72.2\%) used clusterwise inference (Table 3). Fifty studies (12.9\%) reported ROI results and 17 studies (4.4\%) reported uncorrected statistics.

Corrections for multiple comparisons were achieved by various theoretical methods (Table 4)-predominantly parametric methods, regardless of inference at cluster or voxel level. Five studies did not mention their theoretical methods, and all of them used FSL software.

\section{Cluster-Defining Primary Threshold (CDT) of Studies Using the Clusterwise Inferential Method}

As mentioned above, 270 studies used clusterwise inference and thus required a CDT. Nearly half of them $(128,47.4 \%)$ defined their CDTs at or more stringent than $p=0.001$ (Table 5). For studies using SPM, AFNI, and FSL, the proportions of
TABLE 3 | Thresholds of statistical significance used by the 338 surveyed studies reporting whole brain results.

\begin{tabular}{|c|c|c|}
\hline Inferential method & $n$ & $\%$ \\
\hline \multicolumn{3}{|c|}{ Cluster-level inference $(n=244)$} \\
\hline Corrected $p=0.05$ & 228 & 93.4 \\
\hline Corrected $p=0.025$ & 1 & 0.4 \\
\hline Corrected $p=0.01$ & 8 & 3.3 \\
\hline Corrected $p=0.001$ & 7 & 2.9 \\
\hline \multicolumn{3}{|c|}{ Voxel-level inference $(n=71)$} \\
\hline Corrected $p=0.05$ & 67 & 94.4 \\
\hline Corrected $p=0.025$ & 1 & 1.4 \\
\hline Corrected $p=0.01$ & 1 & 1.4 \\
\hline Corrected $p=0.005$ & 1 & 1.4 \\
\hline Corrected $p=0.001$ & 1 & 1.4 \\
\hline \multicolumn{3}{|c|}{ Threshold free cluster enhancement $(n=7)$} \\
\hline Corrected $p=0.05$ & 7 & 100.0 \\
\hline \multicolumn{3}{|c|}{ Uncorrected inference $(n=16)$} \\
\hline$p=0.05, k=40$ & 1 & 6.3 \\
\hline$p=0.005, k=50$ & 1 & 6.3 \\
\hline$p=0.005, k=20$ & 1 & 6.3 \\
\hline$p=0.005, k=10$ & 1 & 6.3 \\
\hline$p=0.005$ & 1 & 6.3 \\
\hline$p=0.001, k=20$ & 4 & 25.0 \\
\hline$p=0.001, k=15$ & 1 & 6.3 \\
\hline$p=0.001, k=10$ & 3 & 18.8 \\
\hline$p=0.001, k=5$ & 1 & 6.3 \\
\hline$p=0.001$ & 2 & 12.5 \\
\hline
\end{tabular}

$k$ means the minimal cluster size expressed in number of voxels.

CDTs reaching this standard were $70.9 \%, 37.6 \%$, and $23.1 \%$, respectively (Figure 1). Eighteen studies (6.7\%) did not report their CDTs. The CDT level did not have a significant correlation with the sample size $\left(r^{2}=0.001, p=0.683\right)$. One of the studies had a sample size of 1,299 subjects, which was much larger than the second-largest sample size at 429 . If this outlier was excluded, there was still no significant correlation $\left(r^{2}=0.007\right.$, $p=0.180)$. 
TABLE 4 | Cross-tabulation of the theoretical methods and statistical thresholds of the 371 surveyed studies reporting corrected statistics.

\begin{tabular}{|c|c|c|c|c|c|c|}
\hline \multirow[t]{2}{*}{ Inferential method } & \multicolumn{5}{|c|}{ Theoretical method } & \multirow[t]{2}{*}{ Total count } \\
\hline & Parametric (FWE) & Parametric (FDR) & Parametric (Monte Carlo) & Permutation & Unknown & \\
\hline Voxelwise & 72 & 18 & & 2 & & 92 \\
\hline Clusterwise & 155 & 12 & 92 & 6 & 5 & 270 \\
\hline TFCE & & & & 9 & & 9 \\
\hline
\end{tabular}

There were 17 studies reporting uncorrected statistics; thus, only 371 studies were included in the table. TFCE, threshold-free cluster enhancement.

TABLE 5 | Cluster-defining primary thresholds (CDTs) of 270 studies using clusterwise inferences.

\begin{tabular}{lcc}
\hline CDT (p-value) & $\boldsymbol{N}$ & $\%$ \\
\hline 0.05 & 9 & 3.3 \\
0.025 & 1 & 0.4 \\
0.02 & 1 & 0.4 \\
0.0125 & 1 & 0.4 \\
0.01 & 60 & 22.2 \\
0.005 & 49 & 18.1 \\
0.001 & 124 & 45.9 \\
0.0004 & 1 & 0.4 \\
0.0001 & 2 & 0.7 \\
0.00001 & 1 & 0.4 \\
Unknown & 21 & 7.8 \\
\hline
\end{tabular}

\section{DISCUSSION}

The updated literature survey reported in this study reaffirmed that clusterwise inference remains the mainstream approach (270/388, 69.6\%) for a cohort of 388 fMRI studies, compared to the previous numbers reported by Carp (2012) (53.2\%), Guo et al. (2014) (63\%), and Woo et al. (2014) (75\%). There were still around 53\% (142/270) studies using clusterwise inference that chose a more liberal CDT than $p=0.001(n=121)$ or did not report their CDT $(n=21)$, down from around $61 \%$ reported in Woo et al. (2014). The ratio of studies reporting uncorrected statistics was much lower than the ratios reported by Carp (2012) (40.9\%), Guo et al. (2014) (19\%), and Woo et al. (2014) (6\%).

With regard to the sample size used in the surveyed studies, the median sample size was 33 . A previous study reported that the median sample size used in the studies published in 2015 was 28.5, based on automated data extraction from Neurosynth ${ }^{1}$ database (Poldrack et al., 2017). It was reassuring that studies using clusterwise inference with smaller sample sizes did not use more liberal CDTs.

In terms of inferential methods, it is still true that FSL studies mainly set their CDTs at $p=0.01$ (default setting of the software), which is more liberal than the $p=0.001$ that was highly recommended by various reports (Woo et al., 2014; Eklund et al., 2016; Roiser et al., 2016). Compared with the articles surveyed by Woo et al. (2014), a similar proportion of FSL studies surveyed in the current report used $p=0.001$ or

${ }^{1}$ neurosynth.org more stringent thresholds (around $23.1 \%$ vs. $20 \%$ ). The falsepositive rate may be influenced by multiple factors, such as the degree of spatial smoothing, experiment paradigm, statistical test performed and algorithms written in the statistical software. Hence, even if the statistical thresholds were set according to recommendations, the rate of false positives could still be high and inhomogeneous across the brain (Eklund et al., 2016). Therefore, some may advocate the use of false-discovery rate (FDR) (Genovese et al., 2002) or non-parametric approaches (Nichols and Holmes, 2002). However, few studies used FDR or non-parametric methods. Potential drawbacks of these methods are that problems may arise when inference is drawn from non-parametric methods (Hupé, 2015), whereas FDR results depend on the probability of non-null effects, which conceptually may not always be valid and different studies may set different thresholds (Hupé, 2015). Regardless of the theoretical methods used, the effect sizes should be reported alongside the brain maps of $p$-values for better comprehension of the results (Wasserstein and Lazar, 2016).

The current study has certain limitations. It would be beneficial to evaluate the effects of altering the statistical thresholds on the outcomes of the surveyed articles. However, it is not possible for a literature survey to achieve this. It should be noticed that the statistical practice is only one of the important aspects of an article. Readers should also evaluate other aspectssuch as methodological details, study power and the flexibility of the analyses. It is important for readers to notice the statistical threshold used for different parts of the results. All of these may influence the quality of an article. Publishing replication studies regardless of statistical significance may help readers better comprehend the data quality (Yeung, 2017). Meanwhile, conducting meta-analysis of functional neuroimaging data can also establish consensus on the locations of brain activation to confirm or refute hypothesis (Wager et al., 2007; Zmigrod et al., 2016; Yeung et al., 2017b,d, 2018).

\section{CONCLUSION}

A considerable amount of studies still used statistical approaches that might be considered as having inadequate control over false positives. There were still around 30\% SPM studies that chose a more liberal CDT than $p=0.01$ or did not report their $\mathrm{CDT}$, in spite of the present recommendations. For FSL studies, it seemed that the CDT practice had no sign of improvement since the survey by Woo et al. (2014). A few studies, as noted in Table 5, chose unconventional 
CDT such as $p=0.0125$ or 0.004 . Such practice might tend to create an impression that the threshold alterations were attempted to show "desired" clusters. As the neuroimaging literature is often highly cited and has continued to grow substantially over the years (Yeung et al., 2017a,c,e), there is a need to enforce a high standard of statistical control over false positives. Meanwhile, the median sample size of the analyzed articles did not differ largely from that of previous surveys, and studies with smaller sample sizes did not use more liberal statistical thresholds. In short, there seemed to be no change in the statistical practice compared to the early 2010 s.

\section{AUTHOR CONTRIBUTIONS}

AY is responsible for all parts of the work.

\section{REFERENCES}

Carp, J. (2012). The secret lives of experiments: methods reporting in the fMRI literature. Neuroimage 63, 289-300. doi: 10.1016/j.neuroimage.2012.07.004

Carter, C. S., Lesh, T. A., and Barch, D. M. (2016). Thresholds, power, and sample sizes in clinical neuroimaging. Biol. Psychiatry Cogn. Neurosci. Neuroimaging 1, 99-100. doi: 10.1371/journal.pone.0184923

Cox, R. W. (1996). AFNI: software for analysis and visualization of functional magnetic resonance neuroimages. Comput. Biomed. Res. 29, 162-173. doi: 10.1006/cbmr.1996.0014

Eklund, A., Nichols, T. E., and Knutsson, H. (2016). Cluster failure: why fMRI inferences for spatial extent have inflated false-positive rates. Proc. Natl. Acad. Sci. U.S.A. 113, 7900-7905. doi: 10.1073/pnas.1602413113

Genovese, C. R., Lazar, N. A., and Nichols, T. (2002). Thresholding of statistical maps in functional neuroimaging using the false discovery rate. Neuroimage 15 , 870-878. doi: 10.1006/nimg.2001.1037

Guo, Q., Parlar, M., Truong, W., Hall, G., Thabane, L., Mckinnon, M., et al. (2014). The reporting of observational clinical functional magnetic resonance imaging studies: a systematic review. PLOS ONE 9:e94412. doi: 10.1371/journal.pone. 0094412

Hupé, J.-M. (2015). Statistical inferences under the Null hypothesis: common mistakes and pitfalls in neuroimaging studies. Front. Neurosci. 9:18. doi: 10. 3389/fnins.2015.00018

Jenkinson, M., Beckmann, C. F., Behrens, T. E., Woolrich, M. W., and Smith, S. M. (2012). FSL. Neuroimage 62, 782-790. doi: 10.1016/j.neuroimage.2011. 09.015

Nichols, T. E., and Holmes, A. P. (2002). Nonparametric permutation tests for functional neuroimaging: a primer with examples. Hum. Brain Mapp. 15, 1-25. doi: 10.1002/hbm.1058

Penny, W. D., Friston, K. J., Ashburner, J. T., Kiebel, S. J., and Nichols, T. E. (2011). Statistical Parametric Mapping: the Analysis of Functional Brain Images. Cambridge, MA: Academic press.

Poldrack, R. A., Baker, C. I., Durnez, J., Gorgolewski, K. J., Matthews, P. M., Munafò, M. R., et al. (2017). Scanning the horizon: towards transparent and reproducible neuroimaging research. Nat. Rev. Neurosci. 18, 115-126. doi: 10. 1038/nrn.2016.167

Roiser, J., Linden, D., Gorno-Tempinin, M., Moran, R., Dickerson, B., and Grafton, S. (2016). Minimum statistical standards for submissions to neuroimage: clinical. Neuroimage Clin. 12, 1045-1047. doi: 10.1016/j.nicl.2016. 08.002

Wager, T. D., Lindquist, M., and Kaplan, L. (2007). Meta-analysis of functional neuroimaging data: current and future directions. Soc. Cogn. Affect. Neurosci. 2, 150-158. doi: 10.1093/scan/nsm015

\section{ACKNOWLEDGMENTS}

The author sincerely thanks Ms. Natalie Sui Miu Wong from Oral and Maxillofacial Surgery, Faculty of Dentistry, The University of Hong Kong for her critical comments and statistical advice.

\section{SUPPLEMENTARY MATERIAL}

The Supplementary Material for this article can be found online at: https://www.frontiersin.org/articles/10.3389/fnhum. 2018.00016/full\#supplementary-material

FILE S1 | The complete lists of the 1,020 articles that were screened initially and the 388 articles that entered the analyses.

Wasserstein, R. L., and Lazar, N. A. (2016). The ASA's statement on p-values: context, process, and purpose. Am. Stat. 70, 129-133. doi: 10.1080/00031305. 2016.1154108

Woo, C.-W., Krishnan, A., and Wager, T. D. (2014). Cluster-extent based thresholding in fMRI analyses: pitfalls and recommendations. Neuroimage 91, 412-419. doi: 10.1016/j.neuroimage.2013.12.058

Yeung, A. W. K. (2017). Do neuroscience journals accept replications? A survey of literature. Front. Hum. Neurosci. 11:468. doi: 10.3389/fnhum.2017.00468

Yeung, A. W. K., Goto, T. K., and Leung, W. K. (2017a). At the leading front of neuroscience: a bibliometric study of the 100 most-cited articles. Front. Hum. Neurosci. 11:363. doi: 10.3389/fnhum.2017.00363

Yeung, A. W. K., Goto, T. K., and Leung, W. K. (2017b). Basic taste processing recruits bilateral anteroventral and middle dorsal insulae: an activation likelihood estimation meta-analysis of fMRI studies. Brain Behav. 7:e00655. doi: 10.1002/brb3.655

Yeung, A. W. K., Goto, T. K., and Leung, W. K. (2017c). A bibliometric review of research trends in neuroimaging. Curr. Sci. 112, 725-734. doi: 10.18520/cs/ v112/i04/725-734

Yeung, A. W. K., Goto, T. K., and Leung, W. K. (2017d). Brain responses to stimuli mimicking dental treatment among non-phobic individuals: a meta-analysis. Oral Dis. doi: 10.1111/odi.12819 [Epub ahead of print].

Yeung, A. W. K., Goto, T. K., and Leung, W. K. (2017e). The changing landscape of neuroscience research, 2006-2015: a bibliometric study. Front. Neurosci. 11:120. doi: 10.3389/fnins.2017.00120

Yeung, A. W. K., Goto, T. K., and Leung, W. K. (2018). Affective value, intensity and quality of liquid tastants/food discernment in the human brain: an activation likelihood estimation meta-analysis. Neuroimage 169, 189-199. doi: 10.1016/j.neuroimage.2017.12.034

Zmigrod, L., Garrison, J. R., Carr, J., and Simons, J. S. (2016). The neural mechanisms of hallucinations: a quantitative meta-analysis of neuroimaging studies. Neurosci. Biobehav. Rev. 69, 113-123. doi: 10.1016/j.neubiorev.2016. 05.037

Conflict of Interest Statement: The author declares that the research was conducted in the absence of any commercial or financial relationships that could be construed as a potential conflict of interest.

Copyright (c) 2018 Yeung. This is an open-access article distributed under the terms of the Creative Commons Attribution License (CC BY). The use, distribution or reproduction in other forums is permitted, provided the original author(s) and the copyright owner are credited and that the original publication in this journal is cited, in accordance with accepted academic practice. No use, distribution or reproduction is permitted which does not comply with these terms. 